\title{
UV Studies Related to the Physico-Chemistry of Planetary and Cometary Environments
}

\author{
M.-C. Gazeau, Y. Benilan, H. Cottin, V. Vuitton, A. Jolly and F. Raulin \\ Laboratoire Interuniversitaire des Systèmes Atmosphériques, UMR \\ CNRS 7583, Universités Paris VII-Paris XII, Créteil, France
}

\begin{abstract}
The complex organic chemistry involved in comets and planetary atmospheres can be better understood via comparison between observations and results obtained by models. In this paper, we point out some lacks in chemical schemes used in models that can be responsible for the discrepancies between observational and theoretical data. Then, we propose UV studies (coupling experimental and theoretical approaches) to be developed in order to determine fundamental parameters: absorption cross sections, quantum yields and rate constants which will be used to improve models as well as to better analyse and interpret observations.
\end{abstract}

\section{Photochemical studies of polyynes - application to Titan's atmo- sphere}

The photochemistry occurring in Titan's $\mathrm{N}_{2} / \mathrm{CH}_{4}$ atmosphere is known to produce many organic compounds. Among them, polyynes $\left(\mathrm{C}_{2 n} \mathrm{H}_{2}\right)$ are of prime interest (Figure 1) since they link the gaseous and the solid phase (normal), they play a role in the balance of molecular/atomic hydrogen control (bold), and they are involved in the indirect dissociation of $\mathrm{CH}_{4}$ that occurs in the stratosphere (italic). Diacetylene $\left(\mathrm{C}_{4} \mathrm{H}_{2}\right)$ has been observed and its relative abundance measured (Hanel 1981) while triacetylene $\left(\mathrm{C}_{6} \mathrm{H}_{2}\right)$ has been detected in laboratory simulations (de Vanssay et al. 1995).

Photochemical models have been developed in order to reproduce the relative abundances and evolution compounds (Yung et al. 1984; Toublanc et al. 1995; Lara et al. 1996). These models failed to provide a quantitative explanation of most of the observational data, suffering from the lack of key parameters in particular at low temperature. We will focus our discussion on the chemical description of acetylene $\left(\mathrm{C}_{2} \mathrm{H}_{2}\right)$ and polyynes photochemistry.

First of all, rate constants are probably responsible for introducing errors in the models. The reactions of the ethynyl radical $\left(\mathrm{C}_{2} \mathrm{H}\right)$ has only been measured for the reaction of $\mathrm{C}_{2} \mathrm{H}$ with $\mathrm{C}_{2} \mathrm{H}_{2}$ (Baulch et al. 1994). In all the current models, the kinetic constants of $\mathrm{C}_{2} \mathrm{H}$ with polyynes are assumed to be the same. Moreover, kinetics of $\mathrm{C}_{2 n} \mathrm{H}$ radicals with $\mathrm{C}_{2} \mathrm{H}_{2}$ are arbitrarily estimated equal to that of the $\mathrm{C}_{2} \mathrm{H}$ with $\mathrm{C}_{2} \mathrm{H}_{2}$ reaction divided by $3 n$. The determination of an accurate value of these parameters at low temperature would contribute to lower the uncertainties in models. 


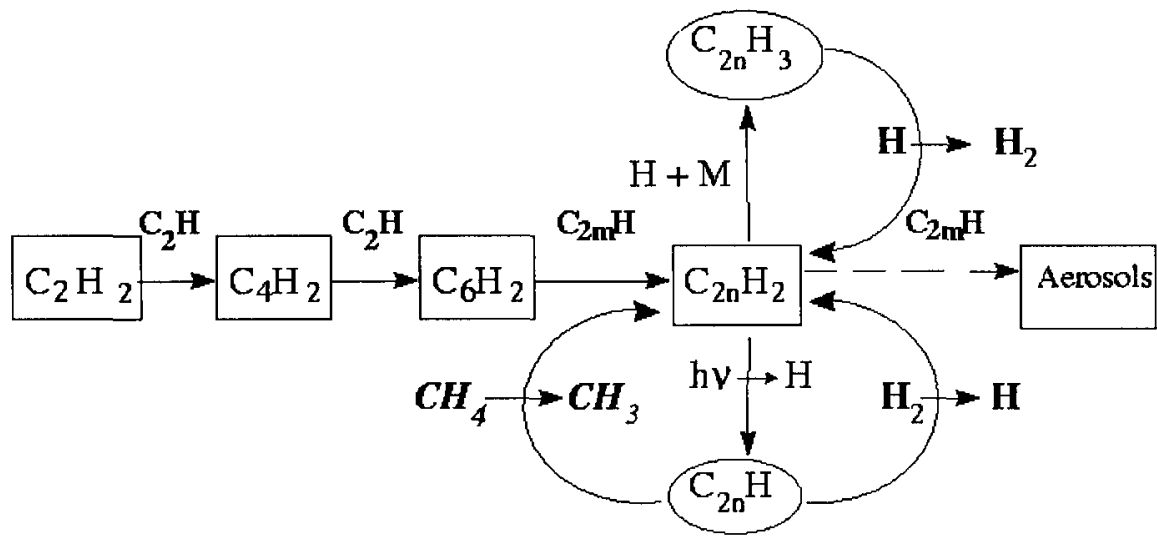

Figure 1. Reaction network for polyynes in Titan's atmosphere.

The dissociation quantum yields are other parameters that present large error bars. It is particularly the case for acetylene: few measurements of $\mathrm{C}_{2} \mathrm{H}$ and $\mathrm{C}_{2}$ radicals production yields from the photolysis of $\mathrm{C}_{2} \mathrm{H}_{2}$ at different wavelengths have been performed (Okabe 1983; Seki and Okabe 1993; Shin and Michael 1991; Satyapal and Bersohn 1991). It has been found that this parameter depends strongly on the irradiation wavelength. This result has to be verified since the ethynyl radical is thought to be involved in the main route leading to the formation of higher polyynes.

Since the dissociation quantum yields of $\mathrm{C}_{2} \mathrm{H}_{2}$ and $\mathrm{C}_{4} \mathrm{H}_{2}$ are low, in some case, a metastable triplet state is supposed to be formed. The latter could compete (if its radiative lifetime is long enough) with radicals to form higher polyynes (Zwier and Allen 1996). Including these processes in the photochemical models could improve the adequation of theoretical and observational data (Lebonnois and Toublanc 2000). Unfortunately, lifetimes of these triplet states are not yet available.

The absorption cross sections depend on the physical conditions such as temperature and pressure. As it is very difficult to reproduce in a laboratory Titan's cold conditions, theoretical calculations are necessary to extrapolate the data obtained at $300 \mathrm{~K}$. For the simple molecule $\mathrm{C}_{2} \mathrm{H}_{2}$, a synthetic spectrum has been built in which temperature and resolution are parameters. The derived absorption cross sections could be included in photochemical models or used to interpret the UV observations (Jolly et al. 1999). Spectroscopic characteristics of highly unstable compounds can be predicted using semi-empirical calculations: as the position and intensities of the bands of $\mathrm{C}_{2 n} \mathrm{H}_{2}$ (up to $n=4$ ) are known, data for longer carbon chains can be determined (Vuitton et al. 2001). 


\section{Photodegradation of high molecular weight compounds - applica- tion to the cometary environment}

It has been established that comets are composed of a mixture of silicate, ices and complex refractory organic molecules (Greenberg and Li 1996). Composition of the coma has been investigated through traditional spectroscopic techniques from the UV-visible to the millimetric and submillimetric range. Observations led to the detection of more than twenty stable small molecules (Crovisier 1998). Laboratory simulation experiments have also enhanced our knowledge about the cometary environment (see the review by Cottin et al. 1999).

At least, four processes are involved in the chemical evolution from the solid phase (nucleus) to the gaseous phase (coma) when the comet is active close to the Sun:

- direct sublimation of a molecule from surface ices. It is typically the case for the $\mathrm{H}_{2} \mathrm{O}$ molecules;

- a "mother" molecule can sublimate and be processed to give a "daughter" molecule in the coma;

- sublimation of a molecule which reacts with a second molecule to form a third one, e.g. the transformation (HCN $\rightarrow \mathrm{HNC}$ ) in comet Hale-Bopp (Irvine et al. 1998);

- small volatile molecules could be released from processed refractory organic material present on grains ejected from the nucleus. This scheme could explain an additional source of carbon monoxide and formaldehyde. The current models failed to reproduce such extended sources. The reason is that some physico-chemical mechanisms are not taken into account, e.g. the photo- or thermo-degradation of the refractory organic component of grains which indeed contribute to the coma's chemical composition. Experimental data concerning such solid-gas processes were missing. However, recently UV irradiations of high molecular weight compounds supposed to be present in the nucleus have been performed. The molecular composition of the resulting gaseous phase has been analyzed and the production quantum yield derived as a function of the wavelength of irradiation (Cottin et al. 2000). Those data are now available and will be introduced in models for polyoxymethylene (P.O.M.) and hexamethylenetetramine (H.M.T.), thought to be "parent" molecules for $\mathrm{HCHO}$ and $\mathrm{CN}$ radicals extended source respectively (Cottin et al. 2001).

\section{References}

Baulch, D. L., Cobbs, C. J., et al. 1994, J. Phys. Chem. Ref. Data 23(6), 847

Cottin, H., Gazeau, M.-C., et al. 1999, Planet. Space Sci., 47, 1141

Cottin, H., Gazeau, M.-C., et al. 2000, J. Photochem. Photobiol., A135, 53

Cottin, H., Bachir, S., et al. 2001, Adv. Space Res., submitted

Crovisier, J., 1998, Faraday Discuss 109, 437

de Vanssay, E., Gazeau M.-C., et al. 1995, Planet. Space Sci., 43, 25

Greenberg, J. M. and Li, A. 1998, Adv. Space Res., 19(7), 981

Hanel, R. 1981, Science, 212, 192

Irvine, W. M., Bergin, E. A., et al. 1998, Nature, 393, 547 
Jolly, A., Benilan, Y., et al. 1999, BAAS, 31, 1150

Lara, L., Lellouch, E., et al. 1996, J. Geophys. Res. 101(E10), 23261

Lebonnois S. and Toublanc, D., 2000, Icarus, in press

Okabe, H., 1983, J. Chem. Phys. 78, 1312

Satyapal, S. and Bersohn, R. 1991, J. Phys. Chem., 95, 8004

Seki, K. and Okabe, H. 1993, J. Chem. Phys., 97, 5284

Shin, K.S. and Michael, J.V. 1991, J. Phys. Chem., 95, 5864

Toublanc, D., Parisot, J.P., et al. 1995, Icarus, 113, 2

Vuitton, V., Scemama, A., et al. 2001, Adv. Space Res., submitted

Yung, Y. L., Allen, M., et al. 1984, ApJS, 55(3), 465

Zwier T. S. and Allen, M. 1996, Icarus, 123, 578 